ISSN 0258-7122

Bangladesh J. Agril. Res. 39(2): 311-336, June 2014

\title{
YIELD AND YIELD ATTRIBUTES OF RAPESEED - MUSTARD (Brassica) GENOTYPES GROWN UNDER LATE SOWN CONDITION
}

\author{
Md. MAhbubul Alam ${ }^{1}$, Ferdousi Begum ${ }^{2}$ AND PRYANKA RoY ${ }^{3}$
}

\begin{abstract}
A field experiment was conducted at the Central Research Station of BARI, Gazipur for two consecutive years 2010-11 and 2011-12 with 30 varieties/ genotypes of rapeseed-mustard under three dates of sowing viz., 25 November, 5 December, and 15 December to determine changes in crop phenology, growth and yield of mustard genotypes under late sown condition when the crop faced high temperature. Days to flowering and maturity were different at different planting times. Date of sowing significantly influenced plant height, siliquae/plant, seeds/siliqua, seed yield, and oil content of seed in both the years. The highest seed yield (1310 and $1535 \mathrm{~kg} / \mathrm{ha}$ ) was obtained from the first planting (25 November) in both the years, which was significantly different from two other dates of sowing. Yield and yield attributes of different varieties varied significantly. Among the varieties, BARI Sarisha-16 of Brassica juncea gave significantly the highest seed yield (1495 and $1415 \mathrm{~kg} / \mathrm{ha}$ ), which was statistically identical to BJDH-11, BJDH-12, BJDH-05, BJDH-20, and BARI Sarisha-6 and significantly different from all other varieties. Interaction effect of variety and sowing date significantly influenced plant height, number of siliquae per plant, number of seeds per siliqua, seed yield, and strover yield. The highest seed yield (1758 and $1825 \mathrm{~kg} / \mathrm{ha}$ ) were recorded from BJDH-11 and BARI Sarisha-16 of Brassica juncea at 25 November planting and BJDH-11 produced the highest yield at 15 December in both the years. The maximum strover yield (3758 and $3825 \mathrm{~kg} / \mathrm{ha}$ ) were obtained from BJDH-11 and BARI Sarisha-16 of Brassica juncea at 25 November planting during 2010-11 and 2011-12. The highest oil content of seeds ( $44.4 \%$ and $45.9 \%$ ) were obtained from the seed of BARI Sarisha-6 and BARI Sarisha-14 at 25 November planting in both the years.
\end{abstract}

Keywords: Late sowing time, genotype, and yield.

\section{Introduction}

Edible oil plays a very important role in human nutrition. As a high-energy component of food, edible oils are important for meeting the calorie requirements. Each gram of oil/fat supplies 9 kilocalories of energy, whereas each gram of carbohydrate/protein furnishes about 4 kilocalories of energy (Stryer, 1980). Fats and oils are also the sources of essential fatty acids. The main

\footnotetext{
${ }^{1 \& 3}$ Scientific Officer, Oilseed Research Centre, Bangladesh Agricultural Research Institute (BARI), Gazipur, ${ }^{2}$ Principal Scientific Officer, Oilseed Research Centre, BARI, Gazipur, Bangladesh.
} 
essential fatty acids of vegetable oils are linoleic and linolenic acids. Fats and oils are used to synthesize phospholipid, which are important components of active tissues viz., brain, nerve, and liver of human beings and other animals. Present dietary pattern of the people of Bangladesh is highly imbalanced due to excess consumption of carbohydrates in the form of cereal more than the body requirement. Carbohydrate contributes about $86 \%$ of the total calorie requirement; on the other hand, fats and oils contribute only about 5-15\% (Ullah, 1989). The average availability of oils and fats in Bangladesh per capita per annum is $3.8 \mathrm{~kg}(10.55 \mathrm{~g} / \mathrm{head} /$ day $)$ against the requirement of $11 \mathrm{~kg}$, while most developed countries consume about $20 \mathrm{~kg}$. Bangladesh has been suffering from acute shortage of edible oil for last several decades. Internal production can meet only about $29 \%$ of our consumption ( $8 \mathrm{~g} /$ day $/$ head). The rest $71 \%$ is met from the import. To meet the annual requirement of edible oil and reduce the import, it is necessary to give immediate attention to increase its domestic production. Increased production of edible oil can come from expansion of cultivated area and in increase production or from both.

Rapeseed-Mustard is a principal oilseed crop, which plays a significant role in the national economy of Bangladesh. But seed yield/ha is very low compared to other rapeseed growing countries of the world, but the productivity of this crop is very low. The low average yield of mustard is due to cultivation of traditional varieties, non-availability of seeds of high yielding varieties and delayed sowing. The genotypes of any crop can express their potential only when grown under optimum management conditions with special emphasis to date of planting. About $20 \%$ yield of mustard reduced due to delayed sowing. There is an ever increasing demand of edible oil in the country and the local production can meet up only one third of the requirement. Every year, it needs to import oil and oilseeds to meet up the deficit. Climatic change may be the major cause for yield reduction in winter crops. Moreover, the rise of global temperatures as predicted between $1.1^{\circ} \mathrm{C}$ and $6.4^{\circ} \mathrm{C}$ during $21^{\text {st }}$ century might aggravate the situation. On the current trends, the average global temperature will rise by $2-3^{\circ} \mathrm{C}$ within next 50 years (Stern, 2006). Temperature is increasing day by day which hampers the growth of rapeseed - mustard and reduced yield. It is cultivated after harvesting of T.aman rice all over Bangladesh. In that case, sowing of mustard is delayed which affect growth and yield of mustard mainly due to rising temperature. Brassica grows well in cool and moist climate (Below $25^{\circ} \mathrm{C}$ day temperature). In general, high temperatures (Higher than $24-25^{\circ} \mathrm{C}$ ) cause a sharp decrease in oil content of most of the seeds. High temperature in Brassica enhanced plant development and caused flower abortion with appreciable loss in seed yield (Rao et al., 1992). Production potentiality of mustard can be fully exploited with suitable agronomic practices and genotypes. It is a fact that a specific genotypes 
does not exhibit the same phenotypic characteristics in all environmental conditions. The different genotypes varied in growth response to different environments and their relative ranking usually differ (Eberhort and Russel, 1966) and ultimately decides the selection of genotypes for a particular or different sowing dates for stabilizing higher yields (Finalay and Wilkinson, 1963; Eberhort and Russel, 1966 and Perkins and Jinks, 1968). Generally, the time of planting varies depending on the climatic condition of the region and the variety to be grown. Different varieties of mustard are sensitive to change in environmental conditions where the crop is being grown. Therefore, it is necessitated to study genotype $\times$ environment interaction to identify varieties stable in different environments (Sarma and Roy, 1993). In general, sowing time of mustard in Bangladesh is confined to a period ranging from mid-October to mid-November (Biswas, K.P. 1989 and Mondal et al., 1999) but with the change of variety and location, various optimums have been suggested (Mondal et al., 1992). Most of the time of a year, temperature is high. So, new varieties must be developed that can withstand adverse climatic condition, particularly high temperature in order to produce increased yield per unit area. Therefore, the present investigation is an attempt to analyze the effect of temperature on seed yield, its component and growth parameters and to identify varieties/genotypes against high temperature.

\section{Materials and Method}

The experiment was conducted at Oilseed Research Centre, Bangladesh Agricultural Research Institute, Joydebpur, Gazipur during the rabi season of 2010-2011 and 2011-12. There were three planting dates $\left(D_{1}=25\right.$ November, $\mathrm{D}_{2}=5$ December, and $\mathrm{D}_{3}=15$ December) and 30 varieties/genotypes of rapeseed/mustard i.e., 11 varieties/genotypes of Brassica napus $\left(\mathrm{V}_{1}=\right.$ Nap-0559, $\mathrm{V}_{2}=$ Nap-0519, $\mathrm{V}_{3}=$ BARI Sarisha- $8, \mathrm{~V}_{4}=$ Nap-0544, $\mathrm{V}_{5}=$ Nap-0538, $\mathrm{V}_{6}=$ Nap$0546, \mathrm{~V}_{7}=$ Nap- 0528, $\mathrm{V}_{8}=$ Nap- 0523, $\mathrm{V}_{9}=$ Nap- 0564, $\mathrm{V}_{10}=$ Nap- 0567and $\mathrm{V}_{11}=$ BARI Sarisha-13), 8 varieties/genotypes from Brassica juncea $\left(\mathrm{V}_{12}=\mathrm{BJDH}-12\right.$, $\mathrm{V}_{13}=$ BJDH-05, $\mathrm{V}_{14}=$ BJDH-11, $\mathrm{V}_{15}=$ BJDH-01, $\mathrm{V}_{16}=$ BJDH-20, $\mathrm{V}_{17}=$ BJDH-17, $\mathrm{V}_{18}=$ BARI Sarisha-11, and $\mathrm{V}_{19}=$ BARI Sarisha-16) and 11 varieties/genotypes from Brssica campestries $\left(\mathrm{V}_{20}=\mathrm{JBC}-5115, \mathrm{~V}_{21}=\mathrm{JBC}-5118, \mathrm{~V}_{22}=\mathrm{BC}-9921, \mathrm{~V}_{23}=\right.$ BC-9917, $V_{24}=$ BC-9922, $V_{25}=$ BC-9909, $V_{26}=$ BARI Sarisha $-9, V_{27}=$ BARI Sarisha $-15, V_{28}=$ BARI Sarisha $-14, V_{29}=$ BARI Sarisha -6 , and $V_{30}=$ Tori-7were used in the experiment. Split plot design was followed in this experiment. The experiment was laid out in split plot design with seeding date in the main plots, and varieties/genotypes in sub-plots. Fertilizer were applied @ 120, 80, 60, 40, 4, $1 \mathrm{~kg} / \mathrm{ha}$ of N, P, K, S, Zn, and B from Urea, TSP, MP, Gypsum, Zinc sulphate, and Boric acid, respectively. Seeds were sown in lines @ 7-8 kg/ha with $30 \mathrm{~cm}$ 
row spacing and continuous seeding in lines. Half of the nitrogen and full amount of other fertilizers were applied at the time of final land preparation. The remaining half of nitrogen fertilizer was applied as top dressing at 22 days after seedling emergence. Weeding cum thinning was done in the third week after sowing. Two irrigations were applied at 22 and 45 days after emergence and plant protection measures were taken whenever necessary. Data were collected on plant height, first flowering, days to maturity, seed yield, and yield attributing characters and oil content in seed. Data were analyzed statistically and treatment means were compared by Least Significant Difference (LSD) test. Weekly maximum and minimum temperature, total rainfall, relative humidity and sunshine hour have also been presented in Appendix (I). The meterological data were collected from Meterological Station at Joydebpur.

\section{Results and Discussion}

\section{Effect of sowing time on yield and yield attributes of mustard}

Different dates of sowing had significant effect on plant height, siliquae/plant, seeds/siliqua, 1000- seed weight. seed yield, stover yield, and oil content (Table 1). Plants of early sowing, flowered later due to prevalence of favourable environment, especially low temperature during vegetative growth phase which enhanced flower initiation in the varieties/lines. Saran and Giri, 1987 also reported that 11 November sowing gave earlier flower. At 25 November sowing, it took 29 days to flower and 87 days to mature and at 15 December sowing, it took 23 to 24 days to flower and 77 days to maturity, respectively (Table 1) in both the years. Delay sowing reduced to flowering and maturity due to higher temperature during late sowing causing reduction in days to flower plants of first planting during early vegetative period and of last planting during later part of the season faced high temperature. Hang and Gilliland (1984) also reported that days to flower and days to maturity varied year to year depending upon the temperature. The highest plant height $(114.4 \mathrm{~cm}$ and $106.8 \mathrm{~cm})$ were found at 25 November sowing time in both the years. The shortest plant $(96.2 \mathrm{~cm}$ and $91.1 \mathrm{~cm}$ ) were found at 15 December sowing time in both the years. In Bangladesh, the average daily temperatures during the third week of October to first week of November $\left(24-27^{\circ} \mathrm{C}\right)$ are quite favourable for quick seed germination and establishment of mustard and rape seedlings (Islam et al., 1994a). Moreover, the sharp fall of both the mean and minimum temperatures from the third week of November onwards shorten the period of inflorescence initiation in mustard and rapes (Islam et al., 1994b) . Mondal and Wright (1986) also found that a temperature range $5-15^{\circ} \mathrm{C}$ is optimum for the normal growth 
and development of rapeseed plant. Above and below this range, temperatures reduced growth rate by reducing plant height and dry matter accumulation. The highest number of siliquae/plant (87.8 and 90.2) were found at 25 November sowing, which was significantly different from that of two other dates of sowing. The lowest number of siliquae/plant (56.3 and 57.3) were found at 15 December sowing. The highest seeds/siliqua (23 and 25), 1000-seed weight (3.4g), and seed yield (1310 and $1510 \mathrm{~kg} / \mathrm{ha}$ ) were obtained from 25 November sowing in both the years. Chakraborty et al. (1991) stated that early sowing produced $24 \%$ higher seed yield than that of later sowing. Delayed planting reduced seed yield drastically. It occured due to rapid inflorescence initiation, severe insect and diseases pest infestation. In late sowing condition, pod number also decreased. Due to high temperatures and long days, pod development phase accelerated rapid maturity of pods and lower yield (Tuteja et al., 1996). The highest oil content in seed $(42.8 \%$ and $43.0 \%)$ were recorded from when seeds sown on 25 November and it was lowest $(41.3 \%$ and $40.6 \%)$ when seeds sown on 15 December (Table 1). Brar et al, (1998) also reported increased seed and oil yield from early planting.

\section{Effect of varieties/genotypes on yield and yield attributes of mustard}

Varieties/genotypes of mustard used in the experiment exerted significant influence on yield and yield attributes (Table 1). Among the 30 varieties/ genotypes, first flowering and maturity days were different and varied from 22 to 34 and 75 to 93 days, respectively. The highest plant height $(165 \mathrm{~cm}$ and $145 \mathrm{~cm})$ was found in BJDH - 05 , which was statistically identical to BJDH -11 and BARI Sarisha-16. Maximum number of siliquae/plant (108 and 90) was recorded in BJDH -05 which differed significantly from other varieties. This has contributed to higher yield. The lowest number of siliquae/plant (52.0 and 56.3) were found in BARI Sarisha-14. The maximum seeds per siliqua (24 and 25) were found from Nap-0538.The highest 1000- grain weight $(3.87 \mathrm{~g}$ and $3.58 \mathrm{~g})$ were obtained from BJDH -17 which was identical to BJDH - 11, 12 and 20 and significantly different from all other varieties due to its bolder seeds. The highest seed yield/ha (1475 and $1415 \mathrm{~kg} / \mathrm{ha}$ ) was obtained from the variety BARI Sarisha -16 and it was due to higher number of siliqua/plant and 1000-seed weight. Similar results was also obtained by Mondal et al. (1992). Podder et al. (1996) also reported that the varieties Daulat and Rai-5 were highly responsive and stable under a wide range of sowing time. The highest oil content of seed (43.4\% and $44.0 \%)$ were obtained from BC-9917 in 2010-11 and BARI Sarisha-14 in 2011-12, which was identical to BARI Sarisha-6, BARI Sarisha-15, BC-9909, BC-9921, and BC9922. The lowest oil content (39.37\% and 40.10\%) was obtained from Nap-0519 and Nap- 0544 in both the years (Table 1). 
Table 1. Effect of sowing date and variety/line on flowering, maturity, growth and yield contributing characters of rapeseed- $\frac{\omega}{\sigma}$ mustard during rabi season of 2010-11 and 2011-12.

\begin{tabular}{|c|c|c|c|c|c|c|c|c|}
\hline \multirow{2}{*}{$\begin{array}{c}\text { Sowing date/ } \\
\text { Genotype }\end{array}$} & \multicolumn{2}{|c|}{ 1st flower (day). } & \multicolumn{2}{|c|}{ Maturity (days). } & \multicolumn{2}{|c|}{ Plant height $(\mathrm{cm})$} & \multicolumn{2}{|c|}{ Siliquae/plant (No.) } \\
\hline & $2010-11$ & 2011-12 & $2010-11$ & 2011-12 & $2010-11$ & 2011-12 & $2010-11$ & 2011-12 \\
\hline November 25 & 29 & 29 & 87 & 87 & 114.4 & 106.8 & 87.8 & 90.2 \\
\hline December 15 & 23 & 24 & 77 & 77 & 96.2 & 91.1 & 56.3 & 57.3 \\
\hline $\mathrm{CV}(\%)$ & - & - & - & - & 7.21 & 5.31 & 11.33 & 9.23 \\
\hline Nap-0559 & 23 & 23.3 & 77 & 77.9 & 79 & 86.5 & 63 & 62.6 \\
\hline Nap-0519 & 24 & 23.2 & 79 & 78.3 & 74 & 87.7 & 52 & 63.1 \\
\hline BARISarisha-8 & 24 & 24.9 & 78 & 78.1 & 87 & 88.9 & 50 & 63.9 \\
\hline Nap-0544 & 23 & 23.7 & 78 & 78.3 & 82 & 84.7 & 47 & 62.2 \\
\hline Nap-0523 & 24 & 24.2 & 79 & 78.8 & 86 & 88.2 & 56 & 63.7 \\
\hline Nap-0564 & 25 & 24.4 & 78 & 78.2 & 86 & 83.7 & 59 & 62.1 \\
\hline Nap-0567 & 25 & 24.4 & 77 & 77.3 & 86 & 83.4 & 55 & 61.7 \\
\hline BARI Sarisha-13 & 25 & 25.9 & 87 & 82.0 & 86 & 82.7 & 58 & 63.5 \\
\hline BJDH-12 & 33 & 33.2 & 94 & 91.8 & 144 & 138.7 & 73 & 87.9 \\
\hline BJDH-05 & 32 & 32.4 & 94 & 93.1 & 160 & 145.2 & 88 & 90.3 \\
\hline
\end{tabular}


Table 1. Cont'd.

\begin{tabular}{|c|c|c|c|c|c|c|c|c|}
\hline \multirow{2}{*}{$\begin{array}{c}\text { Sowing date/ } \\
\text { Genotype }\end{array}$} & \multicolumn{2}{|c|}{ 1st flower (day). } & \multicolumn{2}{|c|}{ Maturity (days). } & \multicolumn{2}{|c|}{ Plant height $(\mathrm{cm})$} & \multicolumn{2}{|c|}{ Siliquae/plant (No.) } \\
\hline & $2010-11$ & 2011-12 & $2010-11$ & $2011-12$ & $2010-11$ & 2011-12 & $2010-11$ & 2011-12 \\
\hline BJDH-11 & 33 & 33.1 & 94 & 92.2 & 148 & 140.0 & 89 & 88.4 \\
\hline BJDH-20 & 33 & 33.0 & 94 & 91.9 & 141 & 139.2 & 88 & 88.0 \\
\hline BJDH-17 & 33 & 32.7 & 93 & 92.0 & 146 & 134.4 & 99 & 86.3 \\
\hline JBC-05115 & 25 & 26.1 & 78 & 82.9 & 84 & 70.4 & 57 & 59.8 \\
\hline JBC-05118 & 26 & 27.0 & 77 & 77.9 & 87 & 73.8 & 47 & 59.5 \\
\hline BC-9921 & 24 & 24.1 & 79 & 78.1 & 99 & 86.2 & 93 & 62.8 \\
\hline BC-9917 & 22 & 22.8 & 78 & 78.1 & 97 & 83.8 & 84 & 61.6 \\
\hline BARI Sarisha-15 & 26 & 24.6 & 76 & 75.9 & 99 & 82.7 & 72 & 61.0 \\
\hline BARI Sarisha-14 & 25 & 23.7 & 77 & 75.8 & 86 & 69.6 & 52 & 56.3 \\
\hline BARI Sarisha-6 & 24 & 26.4 & 81 & 79.1 & 107 & 94.9 & 60 & 66.8 \\
\hline Tori-7 & 22 & 22.0 & 75 & 74.8 & 88 & 85.0 & 93 & 60.6 \\
\hline $\mathrm{CV}(\%)$ & - & - & - & - & 5.79 & 4.58 & 11.33 & 9.33 \\
\hline $\operatorname{LSD}(.05)$ & - & - & - & - & 12.06 & 11.03 & 9.38 & 7.56 \\
\hline
\end{tabular}


Table 1. Cont'd.

$\stackrel{\omega}{\infty}$

\begin{tabular}{l|c|c|c|c|c|c|c|c}
\hline \multirow{2}{*}{$\begin{array}{c}\text { Sowing date/ } \\
\text { Genotype }\end{array}$} & \multicolumn{2}{c|}{ Seeds/siliqua (No.) } & \multicolumn{2}{c|}{ 1000-seed wt $(\mathrm{g})$} & \multicolumn{2}{c|}{ Seed yield (kg/ha) } & \multicolumn{3}{c}{ Oil (\%) } \\
\cline { 2 - 9 } & $2010-11$ & $2011-12$ & $2010-11$ & $2011-12$ & $2010-11$ & $2011-12$ & $2010-11$ & $2011-12$ \\
\hline November 25 & 23 & 83.0 & 3.4 & 3.40 & 1310 & 1535 & 42.8 & 43.0 \\
December 5 & 20 & 77.8 & 3.02 & 2.98 & 1074 & 1201 & 42.0 & 41.8 \\
December 15 & 11 & 110.1 & 2.82 & 2.82 & 816 & 895 & 41.3 & 40.6 \\
\hline CV (\%) & 8.0 & 7.40 & 8.02 & 6.03 & 14.71 & 12.61 & 3.00 & 2.95 \\
LSD(.05) & 7.7 & 6.20 & 0.63 & 0.57 & 103.5 & 99.5 & 1.09 & 1.02 \\
\hline Genotype & & & & & & & & \\
Nap-0559 & 19 & 20.0 & 3.17 & 3.15 & 1157 & 1368 & 41.53 & 41.7 \\
Nap-0519 & 15 & 16.8 & 3.17 & 3.13 & 1020 & 1146 & 39.37 & 41.1 \\
BARI Sarisha-8 & 21 & 22.8 & 3.17 & 3.18 & 1078 & 1341 & 42.57 & 43.2 \\
Nap-0544 & 18 & 19.9 & 3.27 & 3.26 & 995 & 1282 & 40.27 & 40.1 \\
Nap-0538 & 24 & 25.1 & 2.97 & 2.94 & 1033 & 1306 & 42.13 & 42.9 \\
Nap-0546 & 22 & 23.0 & 3.00 & 2.97 & 1080 & 1338 & 42.23 & 42.7 \\
Nap-0528 & 23 & 24.0 & 3.27 & 3.29 & 1076 & 1377 & 42.23 & 42.5 \\
Nap-0523 & 23 & 24.2 & 2.93 & 2.87 & 943 & 1262 & 42.03 & 42.0 \\
Nap-0564 & 23 & 24.3 & 3.00 & 3.05 & 985 & 1274 & 41.67 & 42.0 \\
Nap-0567 & 23 & 24.0 & 3.33 & 3.16 & 838 & 1125 & 41.60 & 41.7 \\
BARI Sarisha-13 & 15 & 16.9 & 3.00 & 2.98 & 848 & 1011 & 41.03 & 42.5 \\
BJDH-12 & 10 & 11.2 & 3.40 & 3.34 & 1187 & 1154 & 40.50 & 38.6 \\
BJDH-05 & 10 & 11.6 & 3.47 & 3.38 & 1307 & 1268 & 40.90 & 38.8 \\
BJDH-11 & 10 & 10.1 & 3.80 & 3.47 & $\mathbf{1 4 9 5}$ & 1408 & 42.70 & 39.1 \\
\hline
\end{tabular}


Table 1. Cont'd

\begin{tabular}{|c|c|c|c|c|c|c|c|c|}
\hline \multirow{2}{*}{$\begin{array}{c}\text { Sowing date/ } \\
\text { Genotype }\end{array}$} & \multicolumn{2}{|c|}{ Seeds/siliqua (No.) } & \multicolumn{2}{|c|}{1000 -seed wt $(\mathrm{g})$} & \multicolumn{2}{|c|}{ Seed yield (kg/ha) } & \multicolumn{2}{|c|}{ Oil (\%) } \\
\hline & $2010-11$ & 2011-12 & $2010-11$ & 2011-12 & $2010-11$ & 2011-12 & 2010-11 & 2011-12 \\
\hline BJDH-01 & 10 & 11.4 & 3.30 & 3.45 & 1252 & 1191 & 42.23 & 40.3 \\
\hline BJDH-17 & 11 & 11.2 & 3.70 & 3.58 & 1272 & 1265 & 41.73 & 39.0 \\
\hline BARI Sarisha-11 & 10 & 10.4 & 2.93 & 3.16 & 1086 & 1195 & 41.43 & 39.4 \\
\hline JBC-05115 & 18 & 19.5 & 3.03 & 3.18 & 921 & 933 & 42.60 & 43.2 \\
\hline JBC-05118 & 20 & 21.9 & 3.13 & 3.15 & 757 & 749 & 42.20 & 43.2 \\
\hline BC-9921 & 17 & 18.9 & 2.40 & 2.70 & 985 & 1133 & 43.23 & 43.6 \\
\hline BC-9917 & 15 & 16.5 & 2.60 & 2.59 & 1071 & 1212 & 43.40 & 43.4 \\
\hline BC-9922 & 14 & 15.2 & 2.70 & 2.66 & 1106 & 1224 & 43.27 & 43.8 \\
\hline BARI Sarisha-14 & 24 & 25.7 & 2.67 & 2.74 & 747 & 975 & 43.00 & 44.0 \\
\hline BARI Sarisha-6 & 16 & 17.7 & 3.37 & 3.07 & 1290 & 1423 & 43.27 & 43.8 \\
\hline Tori-7 & 16 & 17.3 & 2.53 & 2.73 & 788 & 1153 & 41.37 & 42.4 \\
\hline $\mathrm{CV}(\%)$ & 9.4 & 10.23 & 7.12 & 6.10 & 11.71 & 12.01 & 3.02 & 4.02 \\
\hline $\operatorname{LSD}(.05)$ & 6.4 & 5.36 & 0.426 & 0.302 & 99.5 & 110.5 & 1.49 & 1.50 \\
\hline
\end{tabular}


Table 2. Interaction Effect of sowing date and variety/line on flowering, maturity, growth and yield contributing characters of rapeseed-mustard during rabi season, 2010-11 and2011-12.

\begin{tabular}{|c|c|c|c|c|c|c|c|c|c|c|c|c|}
\hline \multirow{3}{*}{$\begin{array}{l}\text { Variety/ } \\
\text { Genotype }\end{array}$} & \multicolumn{6}{|c|}{ 1st Flower(day). } & \multicolumn{6}{|c|}{ Maturity(day). } \\
\hline & \multicolumn{2}{|c|}{25 Nov. } & \multicolumn{2}{|c|}{5 Dec. } & \multicolumn{2}{|c|}{15 Dec. } & \multicolumn{2}{|c|}{25 Nov. } & \multicolumn{2}{|c|}{5 Dec. } & \multicolumn{2}{|c|}{15 Dec. } \\
\hline & $2010-11$ & 2011-12 & 2010-11 & 2011-12 & 2010-11 & 2011-12 & $2010-11$ & 2011-12 & $2010-11$ & $2011-12$ & $2010-11$ & $2011-12$ \\
\hline Nap-0559 & 25.5 & 25.3 & 23.0 & 23.7 & 20 & 21.0 & 84.5 & 86.7 & 76.0 & 76.0 & 71.0 & 71.0 \\
\hline Nap-0519 & 26.5 & 25.3 & 24.0 & 23.3 & 21 & 21.0 & 85.0 & 86.0 & 77.5 & 76.0 & 73.0 & 73.0 \\
\hline BARI Sarisha-8 & 27.0 & 28.3 & 24.0 & 24.7 & 21 & 21.7 & 85.0 & 84.7 & 77.0 & 77.5 & 72.0 & 72.0 \\
\hline Nap-0544 & 26.0 & 26.0 & 23.0 & 24.7 & 20 & 20.3 & 84.0 & 85.0 & 77.5 & 77.0 & 73.0 & 73.0 \\
\hline Nap-0538 & 27.5 & 26.7 & 25.0 & 23.3 & 22 & 22.3 & 86.0 & 85.7 & 78.5 & 77.0 & 74.0 & 74.0 \\
\hline Nap-0546 & 28.5 & 27.0 & 26.0 & 25.3 & 23 & 22.7 & 85.5 & 85.0 & 77.5 & 78.0 & 73.0 & 73.0 \\
\hline Nap-0528 & 28.5 & 26.3 & 26.0 & 25.3 & 23 & 22.7 & 85.5 & 85.3 & 77.5 & 77.0 & 73.0 & 73.0 \\
\hline Nap-0523 & 26.5 & 27.0 & 24.0 & 24.3 & 21 & 21.3 & 86.0 & 86.3 & 77.5 & 77.0 & 73.0 & 73.0 \\
\hline Nap-0564 & 28.0 & 26.3 & 25.0 & 24.7 & 22 & 22.3 & 84.5 & 85.7 & 76.5 & 77.0 & 72.0 & 72.0 \\
\hline Nap-0567 & 28.0 & 26.3 & 25.0 & 24.7 & 22 & 22.3 & 85.0 & 85.0 & 76.0 & 76.0 & 71.0 & 71.0 \\
\hline BARI Sarisha-13 & 28.0 & 28.3 & 25.0 & 26.3 & 22 & 23.0 & 87.0 & 85.0 & 89.5 & 76.0 & 85.0 & 85.0 \\
\hline BJDH-12 & 35.5 & 35.3 & 33.0 & 34.0 & 30 & 30.3 & 99.0 & 97.3 & 93.5 & 89.0 & 89.0 & 89.0 \\
\hline BJDH-05 & 35.0 & 34.7 & 32.0 & 32.7 & 29 & 30.0 & 99.0 & 97.3 & 93.5 & 93.0 & 89.0 & 89.0 \\
\hline BJDH-11 & 36.0 & 35.7 & 33.0 & 33.3 & 30 & 30.3 & 99.5 & 96.7 & 93.5 & 93.0 & 89.0 & 87.0 \\
\hline BJDH-01 & 34.0 & 34.7 & 31.0 & 32.3 & 28 & 28.3 & 97.5 & 96.0 & 92.0 & 93.0 & 87.0 & 90.0 \\
\hline BJDH-20 & 35.5 & 35.3 & 33.0 & 33.3 & 30 & 30.3 & 98.5 & 96.7 & 94.0 & 92.0 & 90.0 & 87.0 \\
\hline BJDH-17 & 35.5 & 35.0 & 33.0 & 32.7 & 30 & 30.3 & 98.5 & 97.0 & 92.5 & 94.0 & 87.0 & 85.0 \\
\hline BARI Sarisha-11 & 34.5 & 34.3 & 32.0 & 32.3 & 29 & 29.3 & 98.5 & 96.0 & 93.0 & 92.0 & 85.0 & 85.0 \\
\hline
\end{tabular}


Table 2. Cont'd.

\begin{tabular}{|c|c|c|c|c|c|c|c|c|c|c|c|c|}
\hline \multirow{3}{*}{$\begin{array}{l}\text { Variety/ } \\
\text { Genotype }\end{array}$} & \multicolumn{6}{|c|}{ 1st Flower(day). } & \multicolumn{6}{|c|}{ Maturity(day). } \\
\hline & \multicolumn{2}{|c|}{25 Nov. } & \multicolumn{2}{|c|}{5 Dec. } & \multicolumn{2}{|c|}{15 Dec. } & \multicolumn{2}{|c|}{25 Nov. } & \multicolumn{2}{|c|}{5 Dec. } & \multicolumn{2}{|c|}{15 Dec. } \\
\hline & 2010-11 & 2011-12 & 2010-11 & 2011-12 & 2010-11 & 2011-12 & 2010-11 & 2011-12 & 2010-11 & 2011-12 & 2010-11 & 2011-12 \\
\hline BARI Sarisha-16 & 36.0 & 36.7 & 33.0 & 34.0 & 30 & 30.3 & 99.5 & 97.0 & 94.0 & 93.0 & 90.0 & 90.0 \\
\hline JBC-5115 & 28.0 & 31.0 & 25.0 & 25.3 & 22 & 22.0 & 80.5 & 81.7 & 79.0 & 94.0 & 73.0 & 73.0 \\
\hline JBC-05118 & 29.0 & 32.7 & 26.0 & 25.7 & 23 & 22.7 & 81.0 & 81.7 & 78.0 & 79.0 & 73.0 & 73.0 \\
\hline BC-9921 & 27.0 & 26.7 & 24.0 & 24.3 & 21 & 21.3 & 84.0 & 83.3 & 79.0 & 78.0 & 73.0 & 73.0 \\
\hline BC-9917 & 25.0 & 26.0 & 22.0 & 22.3 & 19 & 20.0 & 82.5 & 82.3 & 78.5 & 79.0 & 73.0 & 73.0 \\
\hline BC-9922 & 25.5 & 25.3 & 23.0 & 23.3 & 20 & 19.7 & 81.0 & 81.3 & 78.0 & 78.0 & 72.0 & 72.0 \\
\hline BC-9909 & 25.5 & 26.0 & 23.0 & 22.7 & 20 & 20.3 & 81.0 & 81.7 & 77.5 & 78.0 & 72.0 & 72.0 \\
\hline BARI Sarisha-9 & 26.5 & 25.7 & 24.0 & 23.7 & 21 & 20.7 & 79.5 & 80.7 & 77.0 & 77.0 & 69.0 & 70.0 \\
\hline BARI Sarisha-15 & 29.0 & 26.7 & 26.0 & 24.7 & 23 & 22.3 & 81.5 & 80.7 & 77.5 & 77.0 & 69.0 & 70.0 \\
\hline BARI Sarisha-14 & 28.0 & 25.3 & 25.0 & 24.3 & 22 & 21.3 & 80.0 & 80.3 & 78.0 & 77.0 & 73.0 & 70.0 \\
\hline BARI Sarisha-6 & 27.0 & 30.7 & 24.0 & 27.3 & 21 & 21.3 & 80.0 & 83.3 & 88.0 & 78.0 & 76.0 & 76.0 \\
\hline Tori-7 & 24.5 & 24.3 & 22.0 & 22.3 & 19 & 19.3 & 77.0 & 77.3 & 76.0 & 76.0 & 71.0 & 71.0 \\
\hline $\mathrm{CV}(\%)$ & - & & - & & - & & - & 86.0 & - & 76.0 & - & 73.0 \\
\hline $\operatorname{LSD}(0.05)$ & - & & - & & - & & - & 84.7 & - & 77.5 & - & 72.0 \\
\hline
\end{tabular}


Table 2. Cont'd.

N

\begin{tabular}{|c|c|c|c|c|c|c|c|c|c|c|c|c|}
\hline \multirow{3}{*}{$\begin{array}{l}\text { Variety/ } \\
\text { Genotype }\end{array}$} & \multicolumn{6}{|c|}{ Plant height $(\mathrm{cm})$} & \multicolumn{6}{|c|}{ Siliqua/plant (No.) } \\
\hline & \multicolumn{2}{|c|}{25 Nov. } & \multicolumn{2}{|c|}{5 Dec. } & \multicolumn{2}{|c|}{15 Dec. } & \multicolumn{2}{|c|}{25 Nov. } & \multicolumn{2}{|c|}{5 Dec. } & \multicolumn{2}{|c|}{15 Dec. } \\
\hline & $2010-11$ & 2011-12 & $2010-11$ & 2011-12 & 2010-11 & $2011-12$ & $2010-11$ & 2011-12 & 2010-11 & $2011-12$ & $2010-11$ & $2011-12$ \\
\hline Nap-0559 & 91.7 & 79.9 & 66.6 & 91.7 & 86.4 & 81.4 & 79.9 & 79.8 & 55.0 & 57.6 & 53.2 & 56.1 \\
\hline Nap-0519 & 82.1 & 71.7 & 69.6 & 95.3 & 85.7 & 82.1 & 71.6 & 80.0 & 49.5 & 56.7 & 33.4 & 42.0 \\
\hline BARISarisha-8 & 94.0 & 84.3 & 81.5 & 91.5 & 88.9 & 86.2 & 63.6 & 71.8 & 40.0 & 52.9 & 45.0 & 47.1 \\
\hline Nap-0544 & 86.9 & 84.2 & 73.6 & 92.0 & 85.3 & 76.9 & 56.1 & 75.5 & 49.1 & 46.3 & 35.4 & 36.0 \\
\hline Nap-0538 & 89.1 & 82.8 & 76.7 & 90.8 & 85.3 & 72.5 & 58.5 & 60.7 & 43.6 & 51.5 & 41.3 & 41.5 \\
\hline Nap-0546 & 100.0 & 84.3 & 72.6 & 96.3 & 87.1 & 75.2 & 61.6 & 61.3 & 56.1 & 48.7 & 42.2 & 41.7 \\
\hline Nap-0528 & 101.7 & 83.5 & 75.5 & 98.0 & 84.3 & 83.9 & 58.0 & 66.9 & 44.8 & 53.3 & 36.6 & 42.5 \\
\hline Nap-0523 & 89.6 & 84.7 & 82.4 & 90.7 & 90.5 & 83.5 & 63.9 & 59.9 & 56.5 & 49.7 & 48.5 & 47.5 \\
\hline Nap-0564 & 97.1 & 85.4 & 74.0 & 89.8 & 82.8 & 78.4 & 73.1 & 68.4 & 55.4 & 58.3 & 48.5 & 47.7 \\
\hline Nap-0567 & 90.2 & 85.4 & 81.1 & 87.0 & 86.1 & 77.2 & 58.1 & 74.8 & 58.9 & 57.2 & 47.5 & 51.7 \\
\hline BARISarisha-13 & 92.1 & 83.9 & 80.5 & 92.5 & 82.7 & 72.9 & 82.3 & 61.6 & 44.7 & 58.9 & 45.7 & 51.1 \\
\hline BJDH-12 & 153.5 & 146.9 & 131.1 & 150.1 & 132.1 & 133.8 & 76.8 & 83.7 & 78.4 & 66.3 & 64.5 & 49.1 \\
\hline BJDH-05 & 174.9 & 154.2 & 150.1 & 153.6 & 148.2 & 133.9 & 111.2 & 79.3 & 79.4 & 78.5 & 72.8 & 73.5 \\
\hline BJDH-11 & 156.0 & 147.8 & 139.1 & 148.1 & 139.5 & 132.3 & 111.5 & 111.1 & 87.8 & 81.6 & 68.0 & 65.1 \\
\hline BJDH-01 & 155.9 & 144.8 & 136.6 & 153.9 & 132.3 & 122.3 & 110.3 & 118.7 & 74.9 & 89.5 & 62.0 & 64.7 \\
\hline BJDH-20 & 160.9 & 139.1 & 124.4 & 144.6 & 141.7 & 131.2 & 107.8 & 112.8 & 87.2 & 77.7 & 68.8 & 70.6 \\
\hline BJDH-17 & 157.7 & 143.9 & 137.6 & 137.9 & 136.4 & 128.9 & 113.4 & 111.1 & 97.7 & 85.2 & 85.0 & 80.9 \\
\hline BARISarisha-11 & 156.2 & 152.1 & 144.1 & 149.8 & 131.5 & 128.5 & 137.1 & 114.6 & 98.5 & 97.5 & 89.2 & 90.7 \\
\hline BARISarisha-16 & 176.9 & 163.7 & 153.6 & 164.1 & 133.1 & 126.6 & 113.3 & 136.5 & 93.1 & 100.4 & 90.3 & 92.3 \\
\hline
\end{tabular}


Table 2. Cont'd

\begin{tabular}{|c|c|c|c|c|c|c|c|c|c|c|c|c|}
\hline \multirow{3}{*}{$\begin{array}{l}\text { Variety/ } \\
\text { Genotype }\end{array}$} & \multicolumn{6}{|c|}{ Plant height $(\mathrm{cm})$} & \multicolumn{6}{|c|}{ Siliqua/plant (No.) } \\
\hline & \multicolumn{2}{|c|}{25 Nov. } & \multicolumn{2}{|c|}{5 Dec. } & \multicolumn{2}{|c|}{15 Dec. } & \multicolumn{2}{|c|}{25 Nov. } & \multicolumn{2}{|c|}{5 Dec. } & \multicolumn{2}{|c|}{15 Dec. } \\
\hline & $2010-11$ & 2011-12 & 2010-11 & $2011-12$ & 2010-11 & 2011-12 & $2010-11$ & 2011-12 & 2010-11 & $2011-12$ & $2010-11$ & 2011-12 \\
\hline JBC-5115 & 90.8 & 81.9 & 80.3 & 82.1 & 66.1 & 62.9 & 69.9 & 114.0 & 55.6 & 94.6 & 45.1 & 46.9 \\
\hline JBC-05118 & 95.9 & 86.8 & 77.5 & 81.7 & 70.9 & 68.7 & 54.1 & 56.6 & 53.7 & 48.8 & 31.8 & 36.8 \\
\hline BC-9921 & 119.5 & 94.9 & 83.9 & 91.3 & 85.1 & 82.1 & 110.9 & 58.6 & 96.3 & 56.1 & 73.1 & 73.3 \\
\hline BC-9917 & 105.8 & 98.3 & 85.4 & 91.5 & 82.9 & 77.0 & 111.4 & 112.1 & 88.3 & 95.7 & 52.3 & 54.1 \\
\hline BC-9922 & 102.6 & 90.3 & 86.7 & 93.3 & 90.5 & 76.9 & 96.5 & 113.7 & 95.2 & 90.5 & 89.9 & 88.1 \\
\hline BC-9909 & 101.5 & 91.0 & 79.1 & 91.5 & 88.1 & 87.2 & 109.7 & 97.1 & 102.0 & 95.7 & 78.7 & 81.8 \\
\hline BARI Sarisha-9 & 101.6 & 95.6 & 85.4 & 85.9 & 82.3 & 77.2 & 117.7 & 113.9 & 85.0 & 103.1 & 61.3 & 64.1 \\
\hline BARI Sarisha-15 & 103.7 & 100.9 & 91.1 & 88.1 & 84.3 & 75.7 & 106.1 & 117.8 & 67.4 & 87.5 & 41.7 & 45.6 \\
\hline BARI Sarisha-14 & 89.5 & 85.1 & 84.3 & 82.5 & 65.9 & 60.3 & 63.1 & 107.3 & 59.0 & 64.7 & 34.1 & 33.9 \\
\hline BARI Sarisha-6 & 118.3 & 102.6 & 99.3 & 105.8 & 102.9 & 75.9 & 77.4 & 110.3 & 67.4 & 58.4 & 35.4 & 36.3 \\
\hline Tori-7 & 95.1 & 87.3 & 81.6 & 91.1 & 83.1 & 80.8 & 109.8 & 76.3 & 103.3 & 67.1 & 67.2 & 66.1 \\
\hline $\mathrm{CV}(\%)$ & - & - & 5.79 & - & - & 4.58 & - & - & 11.33 & - & - & 9.33 \\
\hline $\operatorname{LSD}(0.05)$ & - & - & 12.06 & - & - & 11.03 & - & - & 9.38 & - & - & 7.56 \\
\hline
\end{tabular}


Table 2. Cont'd.

\begin{tabular}{|c|c|c|c|c|c|c|c|c|c|c|c|c|}
\hline \multirow{3}{*}{$\begin{array}{l}\text { Variety/ } \\
\text { Genotype }\end{array}$} & \multicolumn{6}{|c|}{1000 -seed wt (g) } & \multicolumn{6}{|c|}{ Seed yield (kg/ha) } \\
\hline & \multicolumn{2}{|c|}{25 November } & \multicolumn{2}{|c|}{5 December } & \multicolumn{2}{|c|}{15 December } & \multicolumn{2}{|c|}{25 November } & \multicolumn{2}{|c|}{5 December } & \multicolumn{2}{|c|}{15 December } \\
\hline & 2010-11 & 2011-12 & 2010-11 & 2011-12 & 2010-11 & $2011-12$ & 2010-11 & 2011-12 & $2010-11$ & $2011-12$ & 2010-11 & 2011-12 \\
\hline Nap-0519 & 3.7 & 3.51 & 2.9 & 3.01 & 2.9 & 2.88 & 1182 & 1450 & 1076 & 1033 & 803 & 757 \\
\hline BARISarisha-8 & 3.7 & 3.63 & 3.0 & 2.97 & 2.8 & 2.93 & 1258 & 1740 & 1197 & 1327 & 779 & 956 \\
\hline Nap-0538 & 3.2 & 3.29 & 2.9 & 2.78 & 2.8 & 2.76 & 1258 & 1662 & 985 & 1164 & 855 & 892 \\
\hline Nap-0546 & 3.4 & 3.42 & 2.8 & 2.79 & 2.8 & 2.71 & 1288 & 1743 & 1097 & 1333 & 855 & 937 \\
\hline Nap-0528 & 3.4 & 3.41 & 3.3 & 3.35 & 3.1 & 3.10 & 1242 & 1750 & 1061 & 1351 & 924 & 930 \\
\hline Nap-0523 & 3.3 & 3.04 & 2.9 & 2.99 & 2.6 & 2.57 & 1264 & 1591 & 818 & 1171 & 748 & 923 \\
\hline Nap-0564 & 3.4 & 3.54 & 2.9 & 2.89 & 2.7 & 2.71 & 1167 & 1687 & 1061 & 1293 & 727 & 842 \\
\hline BJDH-05 & 3.7 & 3.53 & 3.4 & 3.43 & 3.3 & 3.17 & 1727 & 1720 & 1233 & 1407 & 961 & 976 \\
\hline BJDH-11 & 4.0 & 3.54 & 3.8 & 3.51 & 3.6 & 3.37 & 1758 & 1708 & 1458 & 1364 & 1268 & 1253 \\
\hline BJDH-01 & 3.5 & 3.86 & 3.3 & 3.37 & 3.1 & 3.14 & 1485 & 1471 & 1348 & 1160 & 924 & 941 \\
\hline BJDH-20 & 3.9 & 3.68 & 3.5 & 3.38 & 3.3 & 3.20 & 1697 & 1669 & 1152 & 1288 & 1112 & 1197 \\
\hline BJDH-17 & 3.9 & 3.83 & 3.7 & 3.50 & 3.5 & 3.41 & 1545 & 1663 & 1348 & 1290 & 924 & 941 \\
\hline BARI Sarisha-11 & 3.1 & 3.54 & 3.0 & 3.00 & 2.7 & 2.94 & 1561 & 1635 & 970 & 1197 & 727 & 753 \\
\hline BARI Sarisha-16 & 3.9 & 3.56 & 3.8 & 3.35 & 3.5 & 3.03 & 1742 & 1825 & 1424 & 1388 & 1258 & 1212 \\
\hline
\end{tabular}


Table 2. Cont'd.

\begin{tabular}{|c|c|c|c|c|c|c|c|c|c|c|c|c|}
\hline \multirow{3}{*}{$\begin{array}{l}\text { Variety/ } \\
\text { Genotype }\end{array}$} & \multicolumn{6}{|c|}{1000 -seed wt (g) } & \multicolumn{6}{|c|}{ Seed yield (kg/ha) } \\
\hline & \multicolumn{2}{|c|}{25 November } & \multicolumn{2}{|c|}{5 December } & \multicolumn{2}{|c|}{15 December } & \multicolumn{2}{|c|}{25 November } & \multicolumn{2}{|c|}{5 December } & \multicolumn{2}{|c|}{15 December } \\
\hline & 2010-11 & 2011-12 & 2010-11 & 2011-12 & 2010-11 & 2011-12 & 2010-11 & 2011-12 & 2010-11 & 2011-12 & 2010-11 & 2011-12 \\
\hline JBC-5115 & 3.3 & 3.63 & 3.1 & 3.02 & 2.7 & 2.90 & 1142 & 1129 & 909 & 925 & 712 & 745 \\
\hline JBC-05118 & 3.4 & 3.34 & 3.1 & 3.15 & 2.9 & 2.95 & 1500 & 1626 & 848 & 712 & 515 & 579 \\
\hline BC-9921 & 2.7 & 3.41 & 2.3 & 2.41 & 2.2 & 2.28 & 1182 & 1450 & 909 & 1162 & 773 & 939 \\
\hline BC-9917 & 3.1 & 3.04 & 2.3 & 2.41 & 2.4 & 2.31 & 1258 & 1740 & 1076 & 1328 & 909 & 959 \\
\hline BC-9922 & 2.9 & 2.93 & 2.5 & 2.42 & 2.7 & 2.63 & 1417 & 1721 & 1152 & 1236 & 909 & 1073 \\
\hline BC-9909 & 3.1 & 2.84 & 2.5 & 2.50 & 2.2 & 2.25 & 1258 & 1662 & 985 & 1065 & 718 & 950 \\
\hline BARI Sarisha-9 & 3.0 & 3.07 & 2.9 & 2.96 & 2.6 & 2.64 & 1288 & 1743 & 955 & 1129 & 909 & 1022 \\
\hline BARI-15 & 3.1 & 3.11 & 2.7 & 2.59 & 2.5 & 2.50 & 1242 & 1750 & 1067 & 1100 & 818 & 891 \\
\hline BARI Sarisha-14 & 3.1 & 3.22 & 2.5 & 2.51 & 2.4 & 2.50 & 1264 & 1591 & 803 & 1094 & 530 & 636 \\
\hline BARI Sarisha-6 & 4.0 & 3.21 & 3.2 & 3.10 & 2.9 & 2.88 & 1167 & 1687 & 1364 & 1284 & 915 & 1038 \\
\hline Tori-7 & 2.9 & 3.45 & 2.5 & 2.52 & 2.2 & 2.23 & 1121 & 1438 & 818 & 1013 & 621 & 714 \\
\hline $\mathrm{CV}(\%)$ & & & 7.12 & & & 6.10 & & & 11.71 & & & 12.01 \\
\hline LSD (0.05) & & & 0.426 & & & 0.302 & & & 99.5 & & & 110.5 \\
\hline
\end{tabular}


Table 2. Interaction effect of sowing date and variety/line on yield contributing characters of rapeseed-mustard during rabi

\begin{tabular}{|c|c|c|c|c|c|c|c|c|c|c|c|c|}
\hline \multirow{2}{*}{$\begin{array}{l}\text { Variety/ } \\
\text { Genotype }\end{array}$} & \multicolumn{6}{|c|}{ Stover yield (kg/ha) } & \multicolumn{6}{|c|}{ Oil (\%) } \\
\hline & $2010-11$ & 2011-12 & 2010-11 & 2011-12 & 2010-11 & 2011-12 & 2010-11 & 2011-12 & 2010-11 & 2011-12 & 2010-11 & 2011-12 \\
\hline Nap-0559 & 3312 & 3626 & 3012 & 3022 & 1758 & 1956 & 42.2 & 43.1 & 41.5 & 41.5 & 40.9 & 40.4 \\
\hline Nap-0519 & 2882 & 3450 & 2576 & 2533 & 1803 & 1757 & 40.2 & 42.3 & 39.2 & 41.0 & 38.7 & 40.1 \\
\hline BARI Sarisha-8 & 3058 & 3740 & 2797 & 3227 & 1779 & 1956 & 42.6 & 43.4 & 43.1 & 43.1 & 42.0 & 42.9 \\
\hline Nap-0538 & 3058 & 3662 & 2085 & 2864 & 1855 & 1892 & 42.6 & 43.2 & 42.3 & 42.9 & 41.5 & 42.5 \\
\hline Nap-0546 & 3027 & 3743 & 2597 & 3233 & 1855 & 1937 & 43.3 & 43.3 & 42.2 & 42.9 & 41.2 & 41.9 \\
\hline Nap-0528 & 3042 & 3750 & 2461 & 3351 & 1924 & 1930 & 43.1 & 44.1 & 42.2 & 42.0 & 41.4 & 41.5 \\
\hline Nap-0523 & 3064 & 3591 & 2018 & 2871 & 1748 & 1923 & 43.2 & 43.8 & 41.7 & 41.7 & 41.2 & 40.6 \\
\hline BJDH-12 & 3742 & 3740 & 3042 & 3033 & 1576 & 1588 & 40.8 & 40.5 & 40.5 & 38.4 & 40.2 & 37.0 \\
\hline BJDH-05 & 3727 & 3720 & 3033 & 3207 & 1961 & 1976 & 41.4 & 40.6 & 40.7 & 38.1 & 40.6 & 37.7 \\
\hline BJDH-11 & 3758 & 3708 & 3258 & 3064 & 3068 & 3053 & 43.5 & 40.0 & 42.4 & 39.2 & 42.2 & 38.2 \\
\hline BJDH-01 & 3485 & 3471 & 3048 & 2960 & 1924 & 1941 & 42.7 & 41.1 & 41.9 & 40.6 & 42.1 & 39.2 \\
\hline BJDH-20 & 3697 & 3669 & 2552 & 3088 & 2512 & 2597 & 42.3 & 41.2 & 42.1 & 39.2 & 40.3 & 37.2 \\
\hline BJDH-17 & 3545 & 3663 & 3348 & 3090 & 1924 & 1941 & 42.8 & 40.2 & 41.7 & 39.2 & 40.7 & 37.6 \\
\hline BARI Sarisha-11 & 3561 & 3635 & 2170 & 2997 & 1727 & 1753 & 42.7 & 40.3 & 41.4 & 39.3 & 40.2 & 38.7 \\
\hline
\end{tabular}


Table 2. Cont'd.

\begin{tabular}{|c|c|c|c|c|c|c|c|c|c|c|c|c|}
\hline \multirow{3}{*}{$\begin{array}{l}\text { Variety/ } \\
\text { Genotype }\end{array}$} & \multicolumn{6}{|c|}{ Stover yield (kg/ha) } & \multicolumn{6}{|c|}{ Oil (\%) } \\
\hline & \multicolumn{2}{|c|}{25 November } & \multicolumn{2}{|c|}{5 December } & \multicolumn{2}{|c|}{15 December } & \multicolumn{2}{|c|}{25 November } & \multicolumn{2}{|c|}{5 December } & \multicolumn{2}{|c|}{15 December } \\
\hline & 2010-11 & 2011-12 & 2010-11 & 2011-12 & 2010-11 & 2011-12 & 2010-11 & 2011-12 & 2010-11 & 2011-12 & 2010-11 & 2011-12 \\
\hline BARI Sarisha-16 & 3742 & 3825 & 3224 & 3288 & 3058 & 3012 & 43.6 & 41.1 & 42.2 & 39.1 & 41.1 & 37.8 \\
\hline JBC-5115 & 2842 & 2929 & 2109 & 1925 & 1712 & 1745 & 43.4 & 44.2 & 42.8 & 43.8 & 41.6 & 41.7 \\
\hline JBC-05118 & 3000 & 3626 & 1848 & 1712 & 1515 & 1579 & 43.1 & 44.1 & 42.0 & 43.7 & 41.5 & 41.8 \\
\hline BC-9921 & 2882 & 3450 & 1909 & 2962 & 1773 & 1939 & 44.2 & 44.6 & 43.0 & 43.8 & 42.5 & 42.5 \\
\hline BC-9917 & 3058 & 3740 & 2476 & 3228 & 1909 & 1959 & 44.2 & 44.8 & 43.1 & 43.8 & 42.9 & 41.7 \\
\hline BC-9922 & 3417 & 3721 & 2852 & 3036 & 1909 & 2573 & 44.2 & 45.0 & 43.1 & 43.7 & 42.5 & 42.8 \\
\hline BC-9909 & 3058 & 3662 & 1985 & 2265 & 1718 & 1950 & 44.2 & 44.8 & 43.1 & 43.6 & 42.7 & 42.9 \\
\hline BARI Sarisha-9 & 3088 & 3743 & 1955 & 2529 & 1909 & 2722 & 43.0 & 44.5 & 42.2 & 43.3 & 41.8 & 41.5 \\
\hline BARI Sarisha-15 & 3042 & 3750 & 2867 & 2600 & 1818 & 1891 & 44.0 & 44.6 & 43.3 & 44.0 & 42.6 & 42.2 \\
\hline BARI Sarisha-14 & 3064 & 3591 & 1803 & 2294 & 1530 & 1636 & 44.0 & 45.9 & 43.1 & 44.3 & 41.9 & 41.7 \\
\hline BARI Sarisha-6 & 2867 & 3687 & 2964 & 3084 & 1915 & 2238 & 44.4 & 45.1 & 43.4 & 44.3 & 42.0 & 42.0 \\
\hline Tori-7 & 2621 & 3438 & 1818 & 2013 & 1621 & 1714 & 42.5 & 44.2 & 41.4 & 42.0 & 40.2 & 41.0 \\
\hline $\mathrm{CV}(\%)$ & - & - & 5.31 & - & - & 6.53 & - & - & 3.02 & - & - & 4.02 \\
\hline $\operatorname{LSD}(0.05)$ & - & - & 302.5 & - & - & 412.3 & - & - & 1.49 & - & - & 1.50 \\
\hline
\end{tabular}


Table 3. Interaction effect of sowing date and variety/line on insect infestation and disease index of rapseed-mustard during the $\underset{\infty}{\sim}$ rabi season, 2010-11 and 2011-12.

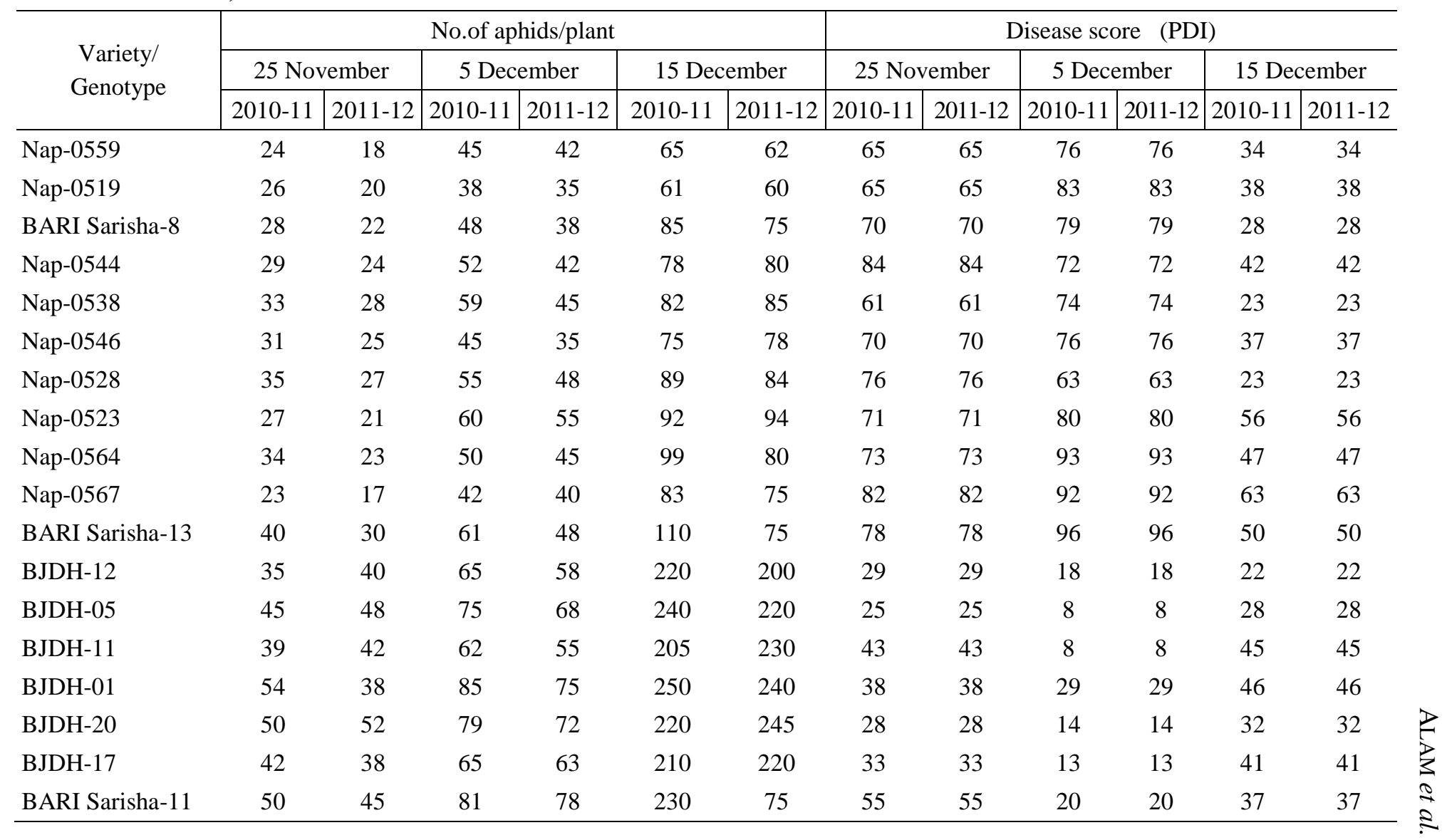


Table 3. Cont'd.

\begin{tabular}{|c|c|c|c|c|c|c|c|c|c|c|c|c|}
\hline \multirow{3}{*}{$\begin{array}{l}\text { Variety/ } \\
\text { Genotype }\end{array}$} & \multicolumn{6}{|c|}{ No.of aphids/plant } & \multicolumn{6}{|c|}{ Disease score (PDI) } \\
\hline & \multicolumn{2}{|c|}{25 November } & \multicolumn{2}{|c|}{5 December } & \multicolumn{2}{|c|}{15 December } & \multicolumn{2}{|c|}{25 November } & \multicolumn{2}{|c|}{5 December } & \multicolumn{2}{|c|}{15 December } \\
\hline & $2010-11$ & 2011-12 & 2010-11 & 2011-12 & $2010-11$ & 2011-12 & 2010-11 & $2011-12$ & $2010-11$ & 2011-12 & 2010-11 & 2011-12 \\
\hline JBC-5115 & 55 & 45 & 95 & 90 & 180 & 200 & 59 & 59 & - & - & 67 & 67 \\
\hline JBC-05118 & 51 & 45 & 87 & 86 & 225 & 235 & 78 & 78 & - & - & 76 & 76 \\
\hline BC-9917 & 50 & 50 & 85 & 98 & 190 & 200 & 50 & 50 & - & - & 44 & 44 \\
\hline BC-9922 & 62 & 42 & 92 & 92 & 210 & 185 & 66 & 66 & - & - & 66 & 66 \\
\hline BC-9909 & 54 & 38 & 85 & 82 & 195 & 200 & 38 & 38 & - & - & 86 & 86 \\
\hline BARI Sarisha-9 & 58 & 32 & 92 & 85 & 215 & 130 & 43 & 43 & - & - & 34 & 34 \\
\hline BARI Sarisha-15 & 41 & 44 & 72 & 65 & 140 & 120 & 37 & 37 & - & - & 23 & 23 \\
\hline
\end{tabular}




\section{Interaction between sowing dates and varieties/ genotypes}

Phenological data of 30 genotypes during three sowing dates are presented in Fig. 1 and 2. Different dates of sowing and varieties/genotypes had significant effect on plant height, siliquae/ plant, seeds/siliqua,1000- seed weight. seed yield, strover yield, and oil content (Table 2).The highest plant height $(176.7 \mathrm{~cm}$ and $163.9 \mathrm{~cm})$ was found in BARI Sarisha-16 sown on 25 November. The shortest plant height $(70.9 \mathrm{~cm}$ and $68.7 \mathrm{~cm})$ was found from 15 December sowing with JBC-05118. On an average, at early sowing it took 26 days and 29 days to flower and 86 and 87 days to mature and at the later sowing, it took 22 and 23 days to flower and 76 days and77 days to maturity, respectively. This variation might have occurred due to rise in temperature. During the months of January and February, temperature started rising which may have been forced the plant to maturity. Hang and Gilliland (1984) reported that days to flower and days to maturity varied year to year depending upon the temperature. Delayed seeding reduced the number of days to flower and days to maturity. Mondal and Wright (1986) also found that a temperature range $5-15^{0} \mathrm{C}$ is optimum for the normal growth and development of rapeseed plant. Above and below this range, temperatures reduced growth rate by reducing plant height and dry matter accumulation. Total dry matter weight $(30.20$ and $33.89 \mathrm{~g}$ ) produced by BJDH-20 genotypes at 25 November sowing (Fig. 1 and 2). The highest number of siliquae/plant (136 and 137) were obtained from 25 November sowing with BARI Sarisha-16 which differed significantly from that of other dates of sowing (Table 2). This happened probably due to comparatively good temperature and moisture received by the plants from 25 November sowing, but high temperature and stressed condition of both these factors was apparent in the later sowing. The lowest number of siliquae/plant (31and36) was found in plant sown at 15 December. The reduction in siliqua on main shoot and seeds/siliqua could be due to floral sterility as temperature $>27^{\circ} \mathrm{C}$ has been reported to induce floral sterility in canola (Morrison and Stewart, 2002) as well as development of flowers into seedless parthenocarpic fruits or flower abortion on the stem due to high temperature (Young et al., 2004). Maximum 1000-seed weight (4.0g) was obtained from BARI Sarisha-6 and BJDH-11 in first year, which was significantly identical to BJDH-01 and BJDH-17 (Table 2) During second year, maximum 1000-seed weight $(3.89 \mathrm{~g})$ was obtained from BJDH-01when plant sown on 25 November. Seed yield showed declining trend in all the varieties under late sown condition. The highest seed yield (1758 and $1825 \mathrm{~kg} / \mathrm{ha}$ ) was recorded from BJDH-11 and BARI Sarisha-16 at 25 November sowing which was identical to BJDH- 05, BJDH-12, BJDH-20, and BARI Sarisha-6 (Table 2). Higher seed yield of BJDH-11 and BARI Sarisha-16 than that of other varieties might be due to higher grain weight. Lowest seed yield (515 and $579 \mathrm{~kg} / \mathrm{ha}$ ) was obtained from JBC-05118 at 15 December sowing which might be due to shorter growing period and thereby less siliquae/plant, 1000-grain weight, and other yield contributing characters. The 


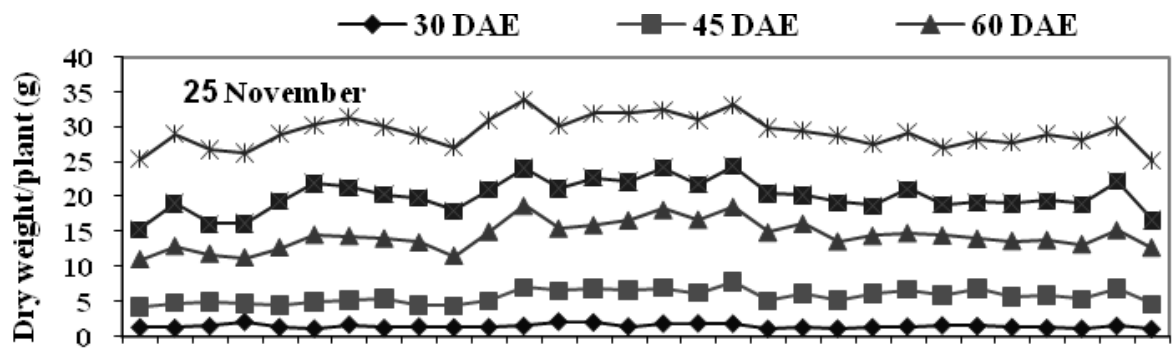

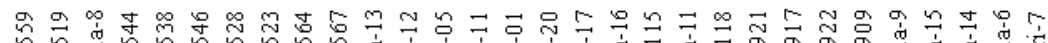

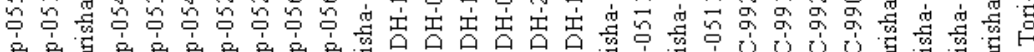

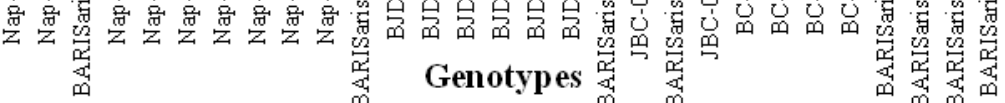
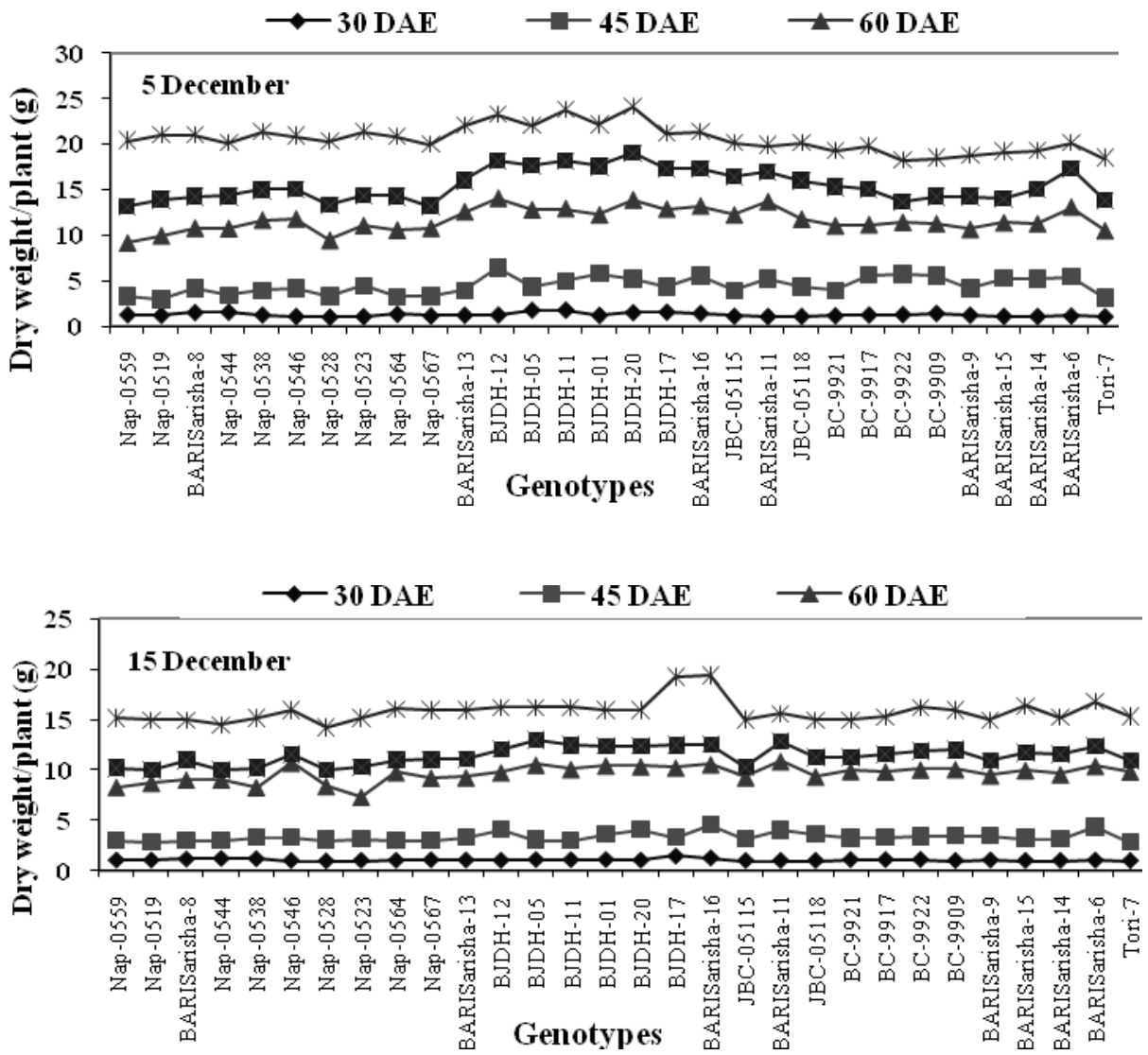

Fig. 1. Total dry matter weight of mustard varieties /genotypes under late sown condition during 2010-11. 

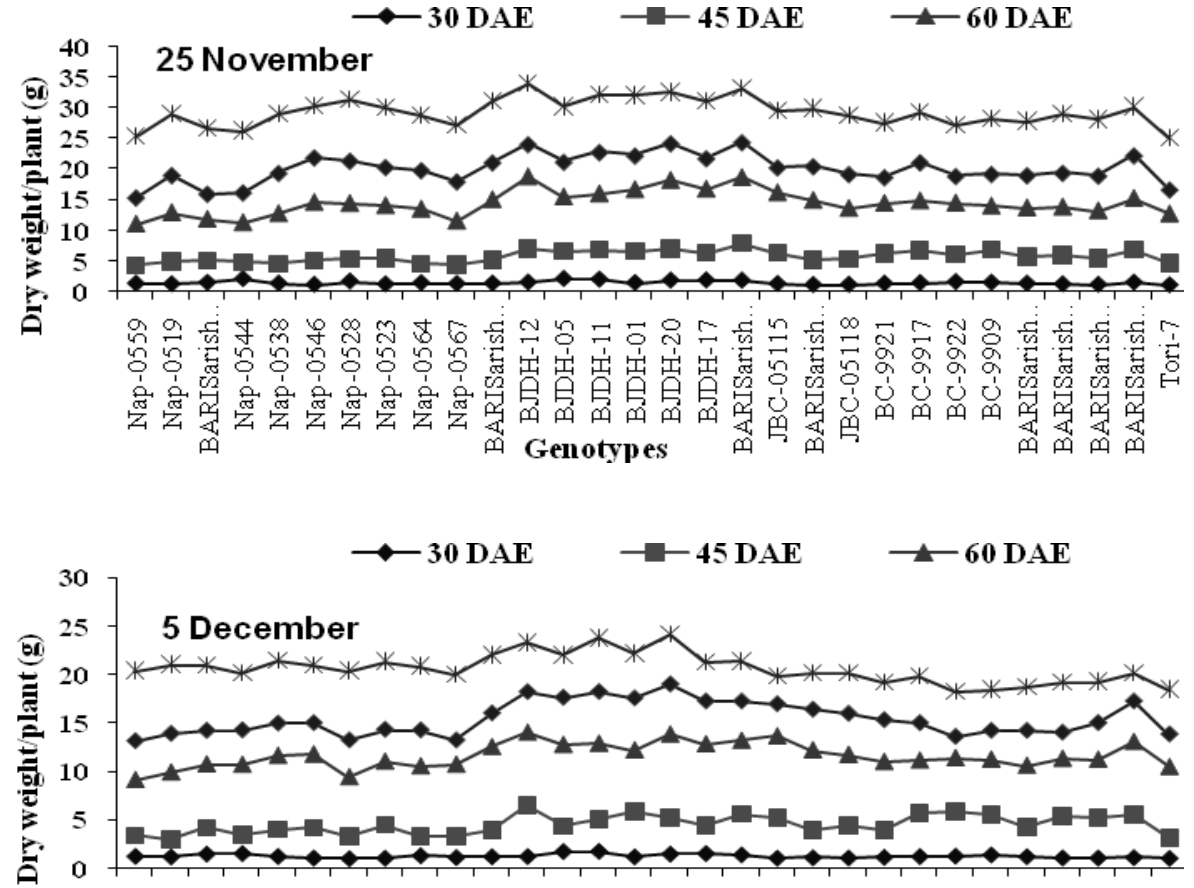

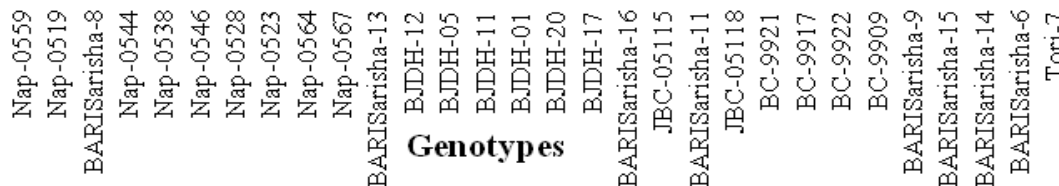
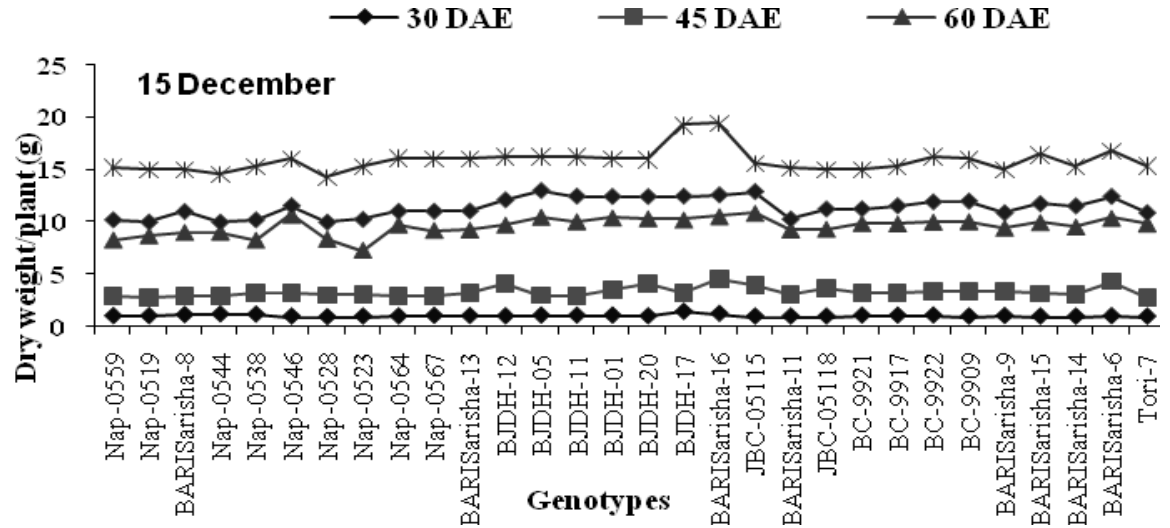

Fig. 2. Total dry matter weight of mustard varieties /genotypes under late sown condition during 2011-12.

results were in agreement with those of Subrahmanyam and Rathore (1994) who observed that high temperature during reproductive stage significantly inhibited the import of photosynthates by both upper and lower pods of terminal reaceme 
and thereby reduced sink strength. Heat stress also decreased oil content as both are negatively correlated. Under late sowing condition, siliqua number also decreased. High temperature and long days during pod development phase accelerated rapid maturity of pods causing lower yield. The maximum stover yield ( 3758 and $3825 \mathrm{~kg} / \mathrm{ha}$ ) was obtained from BJDH-11 and BARI Sarisha-16 of Brassica juncea from 25 November planting. The highest oil content in seed (44.4\% and $45.1 \%$ ) was recorded from BARI Sarisha-6 in 25 November sowing and the lowest oil content in seed $(38.1 \%$ and $39.6 \%)$ was recorded from JBC05118 at 15 December sowing. Insect infestation and disease index were increased with delayed sowing (Table 3). On an average, minimum insect infestation was obtained from first sowing. Maximum insect infestation was obtained from delay sowing due to high temperature which creates favourable condition for insect generation (Table 3). On an average, minimum disease score was found in seeds sown in 25 November and maximum disease score was found in seeds sown in 15 December (Table 3). Brassica juncea group and B. napus group showed better resistant performance against insect infestation and disease than $B$. campestries group. Under late sowing condition, yield performance of $B$. juncea group was better than $B$. campestries and B. napus groups. The five varieties/lines, namely BARI Sarisha -11, 16, BJDH-11, 12, 17, and 20 were adaptable in a wide range of sowing dates. Nap-0559 and Nap- 0546 lines of B.napus groups and BC-9921 and BARI Sarisha-6 of B. campestries were moderately adapted in a wide range of sowing time but $B$. napus groups and $B$. campestries were highly responsive and unstable under a wide range of sowing time. Therefore, these groups were suitable only under the most favourable date of sowing (25 November).

\section{Conclusion}

Form the above results, it may be concluded that the variety BARI Sarisha -11 , BARI Sarisha -16, BJDH-11, 12, 17, and 20, Nap- 0559, BARI Sarisha-6, BC9921, and BC-9922 performed better in 25 November sowing and a wide range of planting time for higher seed yield and delay in sowing reduces seed yield. BARI Sarisha -16, BJDH-11, 12, 17, and 20, Nap-0559, BARI Sarisha-6 and BC-9922 performed better in 5 December sowing and BARI Sarisha -16, BJDH-11,12, and 20 performed better in 15 December sowing. The variety BARI Sarisha-16 may be recommended for late sowing condition after harvest of $\mathrm{T}$. ama rice.

\section{References}

Brar, Z. S., D. S. Bal and A. S. Johi. 1998. Influence of sowing dates,nitrogen and planting geometry on the performance of gobhi sarson (B. napus). Indian $J$. Agronomy 43 (1) 133-137.

Biswas, K. P. 1989. Effect of sowing time on the yield and yield attributes of two mustard varieties. Bangladesh J. Agric. 14 (4): 317-320. 
Chakraborty, P. K., A. Majumdar and B. N.Chatterjee. 1991. Physiological process in Indian mustard Brassica juncea)and yellow sarson (Brassica napus var Glauca) and their agronomic appraisal in mild and short winter prevailing in Gangetic plains of eastern India. Indian J. Agric. Sci. 61(11): 851- 858.

Eberhort, S. A. and W. A., Russel.1966. Stability parameters for comparing varieties. Crop Sci. 6: 36-40.

Finalay, K. W. and G. N.Wilkinson. 1963. The analysis of adaptation in plant breedingprogramme. Aust. J. Agric. Res. 4: 742-754.

Hang, A.N. and G.C. Gilliland. 1984. Planting date effects on yield and quality of oilseed Brassica spp. Washington State University Agric. Res. Centre Res. Bull. 944: 1-12.

Islam, N., O. M. Choudhury., N. A. Sarder and S.M.R. Karim. 1994a. Effects of sowing date on plant development, growth and yield of mustard and rapes-A review. Bangladesh J. Agril Sci. 21: 311-322.

Islam, N., O.M. choudhury., N. A. Sarder and S.M.R. Karim. 1994b. Effects of sowing date on, growth and development of mustard and rapes. Prog. Agric. 5(1): 23-29.

Mondal, M.R.I and M.J. Wright. 1989. Environmental effect on plant growth, morphological characters, yield, oil content and fatty acid composition of canola rapeseed. M.S. Thesis, Cornell University, Ithaca, New York, U.S.A

Mondal, M.R.I., M.A. Islam and M.A. Khaleque. 1992. Effect of variety and planting date on the yield performance of mustard / rapeseed. Bangladesh J. Agril. Sci. 19 (2): 181-186.

Mondal, M.R.I., M.Biswas, M.M. Hydar-Ali and M.A. Akbar. 1999. Response of rapeseed genotype ' Dhali' to seed rate and seeding date. Bangladesh J. Agril. Res. 24(1): 83-90.

Mondal, M. R. I. and M.A.Wahhab. 2001. Production technology of oilcrops. Oilseed Research Centre, BARI, Joydebpur, Gazipur, Bangladesh. Pp. 4 -30.

Morrison, M.J. and D.W. Stewart. 2002. Heat stress during flowering in Summer Brasica. Crop Science 42: 797-803.

Perkins, J. M. and J. L. Jinks.1968. Environmental and genotype environmental components of variability. Heredity 23: $339-356$.

Podder, P., .M. Kader., B.K.Biswas. , M.S. Alam and M.R.Amin 1996. Stability analysis for seed yield and yield components in mustard under different dates of sowing. Bangladesh J. Agril. Sci. 23(2): 1-6.

Rao, G. U., A. Jain and K.T. Shivana. 1992. Effect of high temperature stress on Brassica pollan:viability, germination and ability to set fruits and seeds. Ann. Bot. (London) 69: 193-198.

Sarma, R.N. and A. Roy. 1993. Phenotypic stability for seed yield and maturity in toria ( Brassica napus var. napus) and Indian mustard (Brassica juncea). Indian J. Agril. Sci. 63(12): 814-817.

Saran, G and G. Giri, 1987. Influence of dates sowing on Brassica species under semiarid rainfed condition of north-east India. Indian.J.Agric.Sci, Camb 108: 561-566. 
Stern, M. 2006. Stern Review, the economics of climate change;United Kingdom. SCC. 2006. Addressing technical Assistance needs for adatation to climate change in vulnerable Commonwealth countries; SCC Data paper $1 ; 26^{\text {th }}$ Commonwealth Parliamentary Conference of Members from small countries; Abuja, Nigeria.

Stryer, L. 1980. Fatty acid metabolism in Biochemistry ( $2^{\text {nd }}$ ed.) Stanford Univ. Freeman Co. New York, San Francisco. P. 385.

Subrahamanyam, D. and V. S. Rathore. 1994. Effect of high temperature on CO2 assimilation and partitioning in Indian mustard. Journal Agronomy and Crop Science 172: 188-193.

Tuteja, S. S., Lakpale, R., Tripathi, R. S. 1996. Effect of date of sowing, nitrogen level and nitrogen splitting on mustard (B. juncea). Advances in Plant Sciences 9(1) 167168.

Ullah, A. 1989. Food consumption pattern of rice growing and rice mustard growing household of pabna district in Bangladesh: A comparative study. M.S. Thesis. Institute of Human Nutrition and Food. UPLB. Philippines.

Young, L. W., R. W. Wilen and P. C. Bonham-Smith. 2004. High temperature stress of Brassica napus during flowering reduces micro and megagametophyte fertility, induce fruit abortion and distrupts seed production. Journal of Experimental Botany. 55: 485-495. 
Appendix I. Weekly average minimum and maximum temperature and total rainfall at BARI, Joydebpur, Gazipur during the period from October to April (2010-11and 2011-12).

\begin{tabular}{|c|c|c|c|c|c|c|}
\hline \multirow{2}{*}{$\begin{array}{l}\text { Month and } \\
\text { Week }\end{array}$} & \multicolumn{4}{|c|}{ Temperature $\left({ }^{0} \mathrm{C}\right)$} & \multicolumn{2}{|c|}{ Rainfall (mm) } \\
\hline & Maximum & Minimum & Maximum & Minimum & & \\
\hline October & \multicolumn{2}{|c|}{$2010-11$} & \multicolumn{2}{|c|}{\begin{tabular}{|c|}
$2011-12$ \\
\end{tabular}} & 2010-11 & 2011-12 \\
\hline $1^{\text {st }}$ week & 32.8 & 25.8 & 29.53 & 27.14 & 10 & 5 \\
\hline $2^{\text {nd }}$ week & 31.4 & 23.8 & 30.08 & 25.55 & 11.14 & 13 \\
\hline $3^{\text {rd }}$ week & 33.6 & 25.2 & 28.54 & 24.91 & 6.14 & 0 \\
\hline $4^{\text {th }}$ week & 31.9 & 22.3 & 26.94 & 21.95 & 0 & 0 \\
\hline \multicolumn{7}{|l|}{ November } \\
\hline $1^{\text {st }}$ week & 31.72 & 21.15 & 25.96 & 20.59 & 0.14 & 0 \\
\hline $2^{\text {nd }}$ week & 31.35 & 19.31 & 26.79 & 19.80 & 0 & 0 \\
\hline $3^{\text {rd }}$ week & 30.0 & 19.12 & 24.54 & 19.30 & 0 & 0 \\
\hline $4^{\text {th }}$ week & 29.00 & 17.68 & 24.53 & 17.74 & 0 & 0 \\
\hline \multicolumn{7}{|l|}{ December } \\
\hline $1^{\text {st }}$ week & 27.95 & 14.17 & 24.41 & 17.60 & 0 & 0 \\
\hline $2^{\text {nd }}$ week & 26.06 & 16.7 & 23.33 & 15.81 & 7.57 & 0 \\
\hline $3^{\text {rd }}$ week & 25.56 & 12.20 & 15.54 & 12.73 & 0 & 0 \\
\hline $4^{\text {th }}$ week & 25.48 & 11.21 & 22.76 & 13.15 & 0 & 0 \\
\hline \multicolumn{7}{|l|}{ January } \\
\hline $1^{\text {st }}$ week & 20.67 & 12.43 & 24.13 & 14.51 & 0 & 4 \\
\hline $2^{\text {nd }}$ week & 20.07 & 10.8 & 23.16 & 12.46 & 0 & 6 \\
\hline $3^{\text {rd }}$ week & 19.32 & 9.22 & 23.85 & 11.91 & 0 & 2 \\
\hline $4^{\text {th }}$ week & 25.51 & 12.09 & 25.64 & 10.58 & 0 & 0 \\
\hline \multicolumn{7}{|l|}{ February } \\
\hline $1^{\text {st }}$ week & 27.68 & 12.87 & 27.71 & 11.96 & 0 & 0 \\
\hline $2^{\text {nd }}$ week & 28.85 & 12.48 & 27.09 & 12.53 & 0 & 0 \\
\hline $3^{\text {rd }}$ week & 27.22 & 17.41 & 28.87 & 13.79 & 0 & 0 \\
\hline $4^{\text {th }}$ week & 29.25 & 14.6 & 31.01 & 14.34 & 0 & 0 \\
\hline \multicolumn{7}{|l|}{ March } \\
\hline $1^{\text {st }}$ week & 31.9 & 15 & 31.99 & 18.61 & 0 & 0 \\
\hline $2^{\text {nd }}$ week & 32.1 & 17.8 & 32.68 & 18.84 & 2.75 & 0 \\
\hline $3^{\text {rd }}$ week & 32.13 & 22.2 & 32.81 & 20.09 & 0 & 0 \\
\hline $4^{\text {th }}$ week & 29.88 & 20.9 & 34.65 & 22.19 & 6.37 & 0 \\
\hline \multicolumn{7}{|l|}{ April } \\
\hline $1^{\text {st }}$ week & 31.37 & 20.76 & 32.26 & 21.99 & 9.25 & 7 \\
\hline $2^{\text {nd }}$ week & 34.01 & 21.85 & 30.53 & 22.10 & 0.1 & 62 \\
\hline $3^{\text {rd }}$ week & 33.17 & 21.43 & 34.78 & 22.10 & 3.5 & 80 \\
\hline $4^{\text {th }}$ week & 32.88 & 21.9 & 34.43 & 22.10 & 8.12 & 14 \\
\hline \multicolumn{7}{|l|}{ May } \\
\hline $1^{\text {st }}$ week & 32.78 & 22.5 & 33.01 & 20.74 & 6.85 & 57 \\
\hline $2^{\text {nd }}$ week & 33.34 & 23.21 & 34.61 & 25.33 & 5.71 & 16 \\
\hline $3^{\text {rd }}$ week & 33.07 & 22.91 & 34.79 & 22.99 & 10.0 & 40 \\
\hline $4^{\text {th }}$ week & 33.11 & 24.42 & 34.44 & 26.68 & 8.2 & 36 \\
\hline
\end{tabular}

